\title{
Illumination optics for solid-state lighting
}

\section{Mark Jongewaard}

Mark P. Jongewaard, "Illumination optics for solid-state lighting," Proc. SPIE 11143, Fifteenth Conference on Education and Training in Optics and

Photonics: ETOP 2019, 111432G (2 July 2019); doi: 10.1117/12.2523874

SPIE Event: Fifteenth Conference on Education and Training in Optics and SPIE. Photonics: ETOP 2019, 2019, Quebec City, Quebec, Canada 


\title{
Illumination optics for solid-state lighting \\ Mark P. Jongewaard* \\ Civil, Environmental and Architectural Engineering, 1111 Engineering Drive \\ UCB 428 |University of Colorado, Boulder, CO 80309-0428
}

\begin{abstract}
The entire field of optics \& photonics is vast, encompassing a wide range of technologies and affecting an even larger range of industries. The niche needed by the lighting industry is illumination optics, and in particular, illumination optics for solid-state devices. Optics for this industry have unique requirements that go beyond the basic achievement of a specific intensity distribution, as they are also often required to homogenize the variable spectral properties of the LEDs and provide a desired luminous appearance. Additionally, they need to fit within specific packaging constraints driven by all types of factors including the overall product aesthetic, manufacturing methods and cost. The styles of optics used to meet these requirements vary widely and are limited only by physics and the imagination of the designer. Instilling the right set of skills in students to succeed in this industry is therefore the challenge. Methods employed to date include a mix of the following:

1. fundamentals of photometry, reflection \& refraction,

2. LED properties and package styles,

3. material properties including light scattering,

4. photometric labs,

5. reflector \& refractor design development \& simulation in software, and

6. optical design prototyping.

Illumination optics are used in a wide range of industries including architecture, roadways, entertainment, automotive, aerospace, marine, medical, and consumer products. While this curriculum is focused on the narrow field of illumination, the skills are applicable to a much broader range of non-imaging applications including UV irradiation for disinfection \& curing, IR sensors, light pipes, CPV, and daylighting.
\end{abstract}

Keywords: illumination optics, LEDs, photometry, non-imaging optics, reflector design, refractor design, solid-state lighting

\section{INTRODUCTION}

This course description is intended to fit in the lighting program curriculum within architectural engineering at the University of Colorado, Boulder. Within architectural engineering, the students choose an area of concentration from building mechanical systems (HVAC), structural design, construction management \& illumination. The illumination curriculum includes illumination 1, illumination 2, luminous radiative transfer, advanced lighting design, daylighting, psychology of perception, theatrical lighting \& optical design for illumination and solid-state lighting. The students from this program generally pursue jobs practicing architectural lighting design or working at lighting equipment manufacturers. The intent of the optical design course is to prepare students to design the optical systems used in the lighting equipment, also referred to as luminaires. The students in this program are engineers, but do not have an indepth optics background, having only had 2 semesters of general physics.

*mark.jongewaard@colorado.edu; phone 1-720-891-0030; web https:/www.colorado.edu/lightingprogram/ 
The approach taken to teach illumination optical design has been to first ensure the students know how light behaves and how it is quantified, photometrically \& radiometrically. This is accomplished through a combination of problem-based assignments and labs where light is measured and derived quantities computed. Optical design experience is gained through a range of design examples that show them various optic styles and design features that they can then apply to other designs that have similar requirements. The final project brings everything together, having them define the desired performance \& geometric constraints and then design, build \& physically test the optic. The class has evolved with the goal of trying to find the right balance of theory \& practice. Engineering students generally prefer working with equipment, building things and having a chance to create beyond just solving problems on paper or in software.

\section{FUNDAMENTALS}

\subsection{Photometry \& Radiometry}

The students do have a background in photometry, but repetition is important to ensure concepts are understood and remembered. Assignments and labs are designed to force students to repeatedly draw upon the photometric fundamentals. Understanding photometry is a critical first step since all luminaire performance will be judged via photometric quantities. It all starts with the definition of the lumen, the fundamental quantity of light, and its relation to radiant watts. All other metrics just consider lumens in different geometric conditions. We cover the following photometric \& radiometric quantities:

1. Lumens / radiant watts

2. Illuminance / irradiance

3. Exitance / radiant exitance

4. Luminous intensity / radiant intensity

5. Luminance / radiance

6. The inverse square cosine law

7. Photometric conventions

8. Near field/far field photometry

\subsection{Colorimetry}

Color metrics play an important role in solid-state lighting since LEDs are available in such a wide range of color options. White light can be generated from phosphor converted blue LEDs or mixing several pure color LEDs. As such, a wide variety of "white" light can be generated, and it needs to be judged for its quality. Color metrics are intended to quantify colors such as warm to cool as well as color quality. Color metrics have limitations however, so it is important to understand how the metrics are computed from spectral power distributions (SPDs). The following color metrics are covered:

1. Tristimulus values (XYZ)

2. Chromaticity values $(\mathrm{x}, \mathrm{y}, \mathrm{z})$

3. Uniform color space chromaticity values (u'v')

4. Color temperature (CCT)

5. Color Rendering Index (CRI)

6. IES TM-30 color fidelity and color gamut metrics 


\subsection{LEDs}

LEDs have unique luminous and spectral properties compared to "traditional" types of lamps. There are also many types of LED packages including single dies on a base, mid-powers with 1 or more dies encased within a phosphor infused silicone matrix, high output chip on boards (COBs) with many chips mounted on a single substrate, remote phosphor modules, etc. White LEDs have complex spectral properties that can change over the emission area as well as over the emission angle. Spectral properties can also change over time. The lumen output has a non-linear relationship to drive current and will change with the running temperature. On top of all of this, LEDs are sold in bins based on their spectral, luminous and electrical properties. These aspects are all covered with particular attention to those areas that affect the optical performance of designs using LEDs.

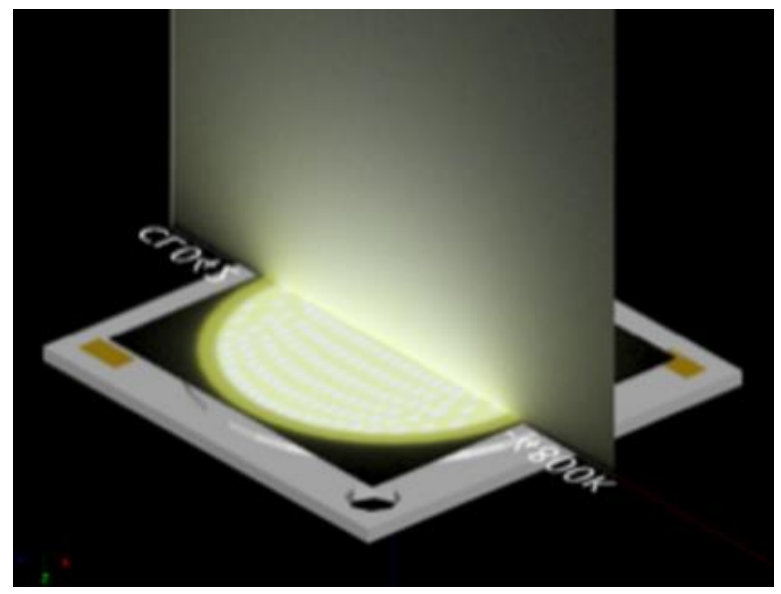

Figure 1. Image of the changing color across the luminous surface as well as over the emission angle for a chip on board (COB) LED package, as simulated in the Photopia optical design software.

\subsection{Luminaire performance criteria}

Lighting equipment is not designed to simply emit light as efficiently as possible. If that was the main goal, then bare lamps would be used since they are by definition $100 \%$ efficient. Lighting equipment generally need to direct light toward a task or target area, light that area with a particular desired quality such as uniformity, and in many cases, the luminaire must have a desired luminous appearance, which could be based on an overall aesthetic or a practical measure such as minimizing glare. The ideal performance varies for every product on the market and even changes over time. Some performance criteria are also defined by organizations such as the DOE Energy Star program or the DesignLights Consortium (DLC). A range of product performance examples are reviewed to show the types of criteria that generally need to be considered.

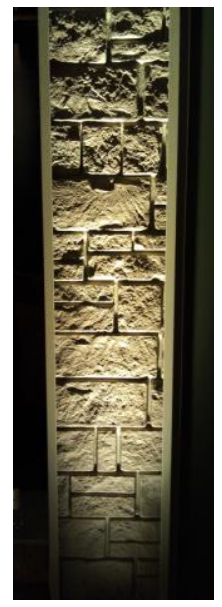

Figure 2. Wall grazer example. Requires a narrowly focused, uniform wash of light from a light source mounted very close to the wall to highlight the $3 \mathrm{D}$ features of a textured surface. 


\subsection{Light control with reflection}

Light control can be done with reflectors or lenses. Each has their own benefits and drawbacks. We start with reflector design methods, which are generally based on developing curves that are comprised of a set of parabolas or ellipses. These sets are directed toward a desired range of aiming angles or points and then their relative sizes are adjusted to control the amount of light directed toward each angle or point. This design method is iterative, but systematic so that the designer can converge on their desired solution. Design concepts start with assumed specular surfaces, but the effects of surface faceting and non-specular finishes are also covered as additional design controls.

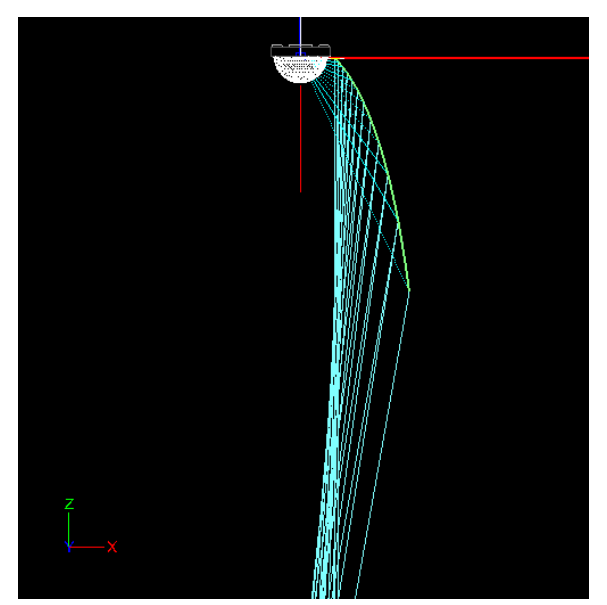

Figure 3. Reflector profile comprised of a set of parabolas aimed toward a range of angles in the beam.

\subsection{Manufacturing methods for reflectors}

Manufacturing methods for reflectors are described and shown via videos so that manufacturing constraints are better understood. If a reflector is to be formed on a spinning chuck for example, then it can't have any undercuts otherwise it could not be pulled off the tool. It is important to always understand how the reflector will be manufactured since that will determine what types of shapes are acceptable as well as the types of surface finishes that can be achieved. Furthermore, different methods have different limitations on part accuracy. Accuracy is certainly important, but the exact precision required depends on the application.

\subsection{Light control with refraction}

The general concepts of refraction via Snell's Law are covered, including TIR conditions. Fresnel's reflections are described so that secondary light interactions in refractor-based optics are understood. Refractive optics can be smooth curves, prismatic and include sections that use direct refraction alongside other sections that utilize TIR. TIR features are useful when light needs to be redirected toward angles beyond what direct refraction can achieve. Lens based optics have become the dominant type of optic for many LED applications since they can capture and therefore control all light emitted by the LED. Whereas reflectors cannot control those lumens that exit the reflector opening directly.

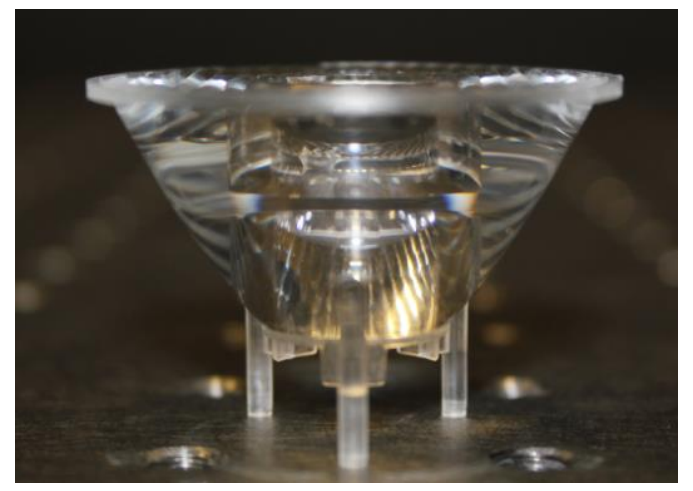

Figure 4. TIR collimator style lens, which includes refractive elements in the center and TIR features around the outside. 


\subsection{Manufacturing methods for refractors}

Refractive optics are manufactured via a range of methods such as injection molding and extrusions if made from plastic. Glass parts are generally cast in molds. As with reflectors, each method includes constraints on the geometry that can be handled, the surface finish and part precision. There are cost trade-offs, so the right combination of part accuracy \& cost needs to be determined based on the specific needs of the application. Most illumination applications don't utilize ground glass lenses as used in imaging optics, since the shapes required for illumination or irradiation are generally not constrained to spherical or aspherical profiles.

\section{LABS}

The lab exercises are designed to emphasize the fundamental photometric quantities and behavior of light and light sources.

\subsection{Measure intensity distributions}

Intensity distribution measurements are based on illuminance measurements and the application of the inverse square cosine law. Measurements are taken in 2 different geometric conditions. The first uses a mirror goniophotometer. The second uses a set of illuminance measurements collected along a wall toward which the light source is aimed. Both methods are manual (no automation) and require unique data collection and data processing methods.

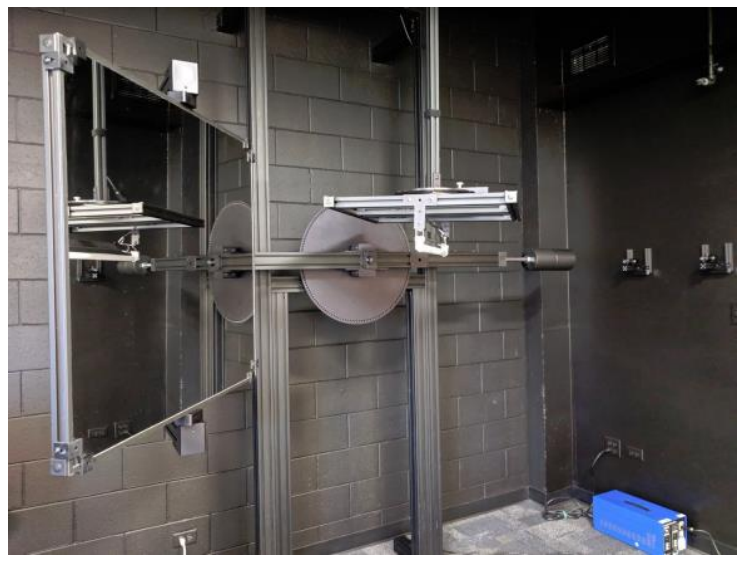

Figure 5. Mirror goniophotometer in the University of Colorado, Boulder lighting lab.

\subsection{Measure LED spectral properties}

LED spectral properties are measured in 2 ways. The first measures the changing spectrum over the emission angle on an optical bench. The second measurement is done in an integrating sphere, capturing the total radiant watts by wavelength emitted by the light source under test. The operating principles of the integrating sphere are covered and the data processing done by the students allows them to compute the source SPD, the total radiant watts, the radiant efficiency, total lumen output and the luminous efficacy. They utilize the equation defining lumens from radiant watts and the photopic efficiency function, directly applying the lumen integration equation. 


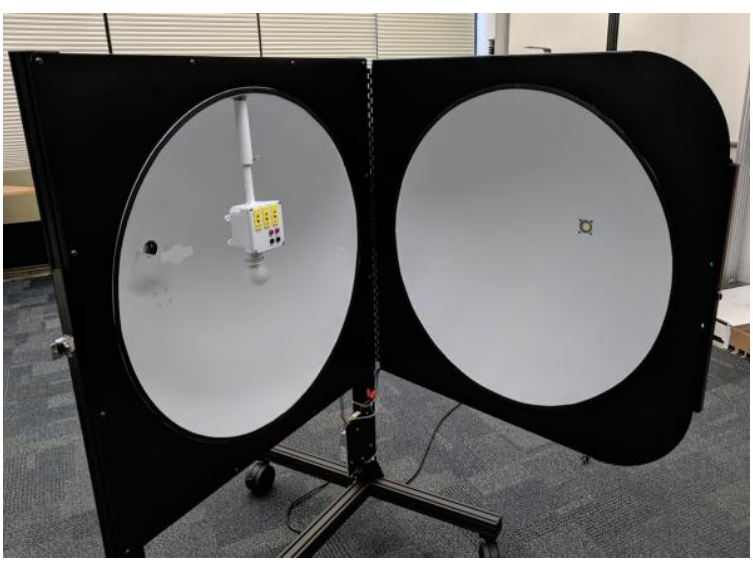

Figure 6. Integrating sphere in the lighting lab.

\subsection{Measure LED output vs temperature, intensity vs distance \& visual photometry}

This lab covers several topics, starting with measurements of LED output as a function of temperature and observing the important role of a heat sink. Near field / far field photometry is explored by computing luminous intensity as a function of the distance at which the illuminance measurements are taken. The students also complete an exercise in visual photometry, which again employs the inverse square cosine law and allows them to determine the relative output of one lamp compared to another all with only a visual comparative method, which was used before the advent of electrical photosensors.

\section{DESIGNS}

A progression of optical designs are pursued with the intension of showing a range of design methods and optic styles. Illumination optical design can sometimes require novel ways of creating optics to achieve a desired behavior, so it is important to gain a range of experiences. Since course time is limited, only a small sample of designs can be pursued, but in order to include as many as possible, the designs and performance simulations are all done in optical design software. The final project does however provide the opportunity to physically build and test their optical design. The students use the Photopia optical design software for this course, and they have access to laser cutters and 3D printers to build their final project prototype.

\subsection{COB floodlight reflectors}

Given a 4000 lumen Bridgelux COB LED, the design goal is to produce a $35^{\circ}$ beam angle with a smooth distribution and good color mixing in a $90 \mathrm{~mm}$ diameter reflector. The first design uses a reflector with a cross aiming scheme and a second version uses a reflector where no light crosses the central axis. The intent being to show how the same problem can be solved multiple ways, which is useful to satisfy other design constraints that could be imposed. A final variation is designed to achieve the narrowest beam possible within this same diameter. 


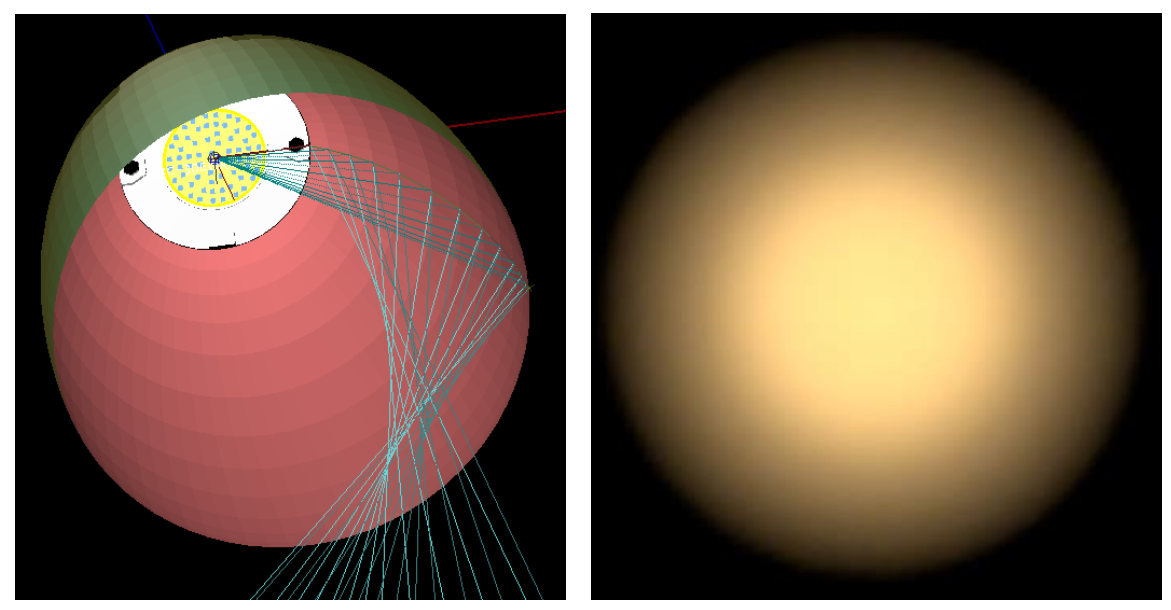

Figure 7. COB LED medium flood faceted reflector design shown on left. The smooth spot produced by the warm white LED with well mixed color on the right.

\subsection{Refraction \& Fresnel reflection spreadsheets}

As an introduction to refractor design, a spreadsheet tool is created that computes the refracted ray direction based on the material index, an incoming ray direction and the tilt angles of the entrance and exit surfaces of a prism. This tool is then used in the following 2 refractor design assignments to determine key angles in their designs. Additionally, a spreadsheet tool is created that computes the reflection and transmission of light entering and exiting a refractor based on the material index, incidence angle of the light and Fresnel's equations.

\subsection{TIR collimator design}

A collimator lens is designed, which uses a central smoothly curved refractive element and an outer surface that reflects via TIR. This design uses both reflector and lens design methods. The central feature design method is simplified for this first design, but still provides the desired effect.
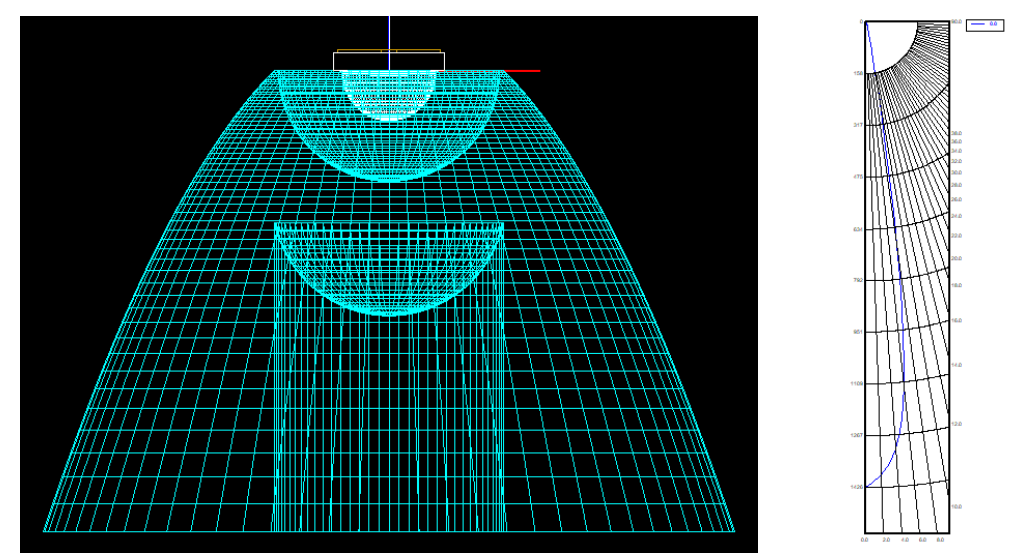

Figure 8. An LED collimator lens design and the polar plot showing its narrow beam intensity distribution.

\subsection{Tubular skylight lens design}

Tubular skylights can include refractive features on their dome cover lens that are intended to improve light collection when the sun is low in the sky while decreasing light collection when the sun is higher in the sky. This is desired so that the minimum required light that enables electric lights to be turned off is provided over a longer portion of the day and so that the lumen collection is more consistent over the course of the day. The spreadsheet tool was used to determine the required prism angles on the dome lens. Photopia was used to model the sun and sky light collected and distributed by the full tubular skylight device under various solar altitude angles, both with and without the prismatic features in order to determine their effectiveness. Daylight device designs are a result of many trade-offs since they need to work for both sun and sky light under a wide range of changing conditions. 

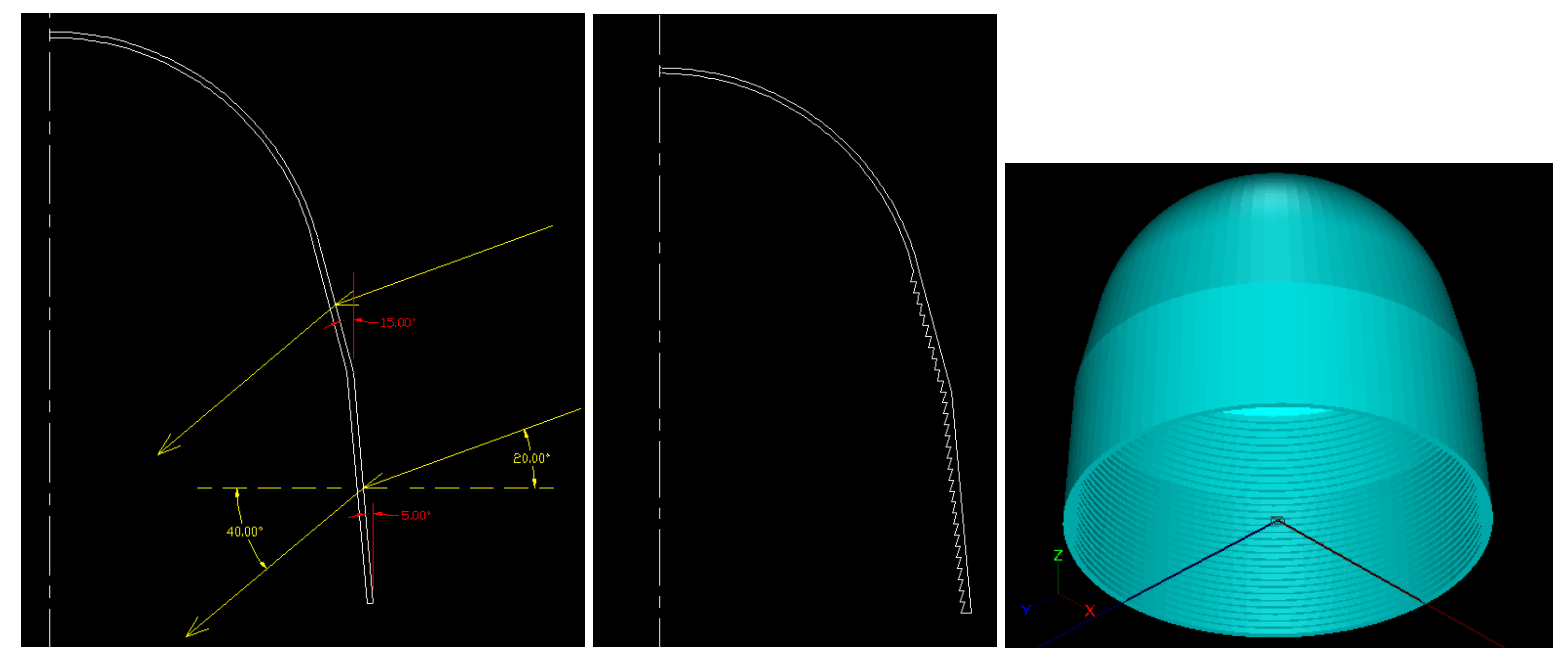

Figure 9. Tubular skylight dome lens profile, showing the desired beam shift for incoming, low angle sunlight in the left image. The central image shows the required prisms added to this profile to redirect the light. The right image shows the full dome lens part ready to be simulated with the tubular skylight system.

\subsection{Wide beam square and forward throw outdoor area lens designs}

Creating a square light pattern requires a lens shape that isn't round or square, but is a "free-form" shape in between. The general method for this design process is introduced and the design is created with more advanced lens design tools learned in Photopia. Understanding this design method also leads the students toward designing more complex "forward throw" optics, such as those required for roadway and area lights. These are some of the most challenging lens design types required in the lighting industry, so it is helpful for students to be introduced to these design methods as well.
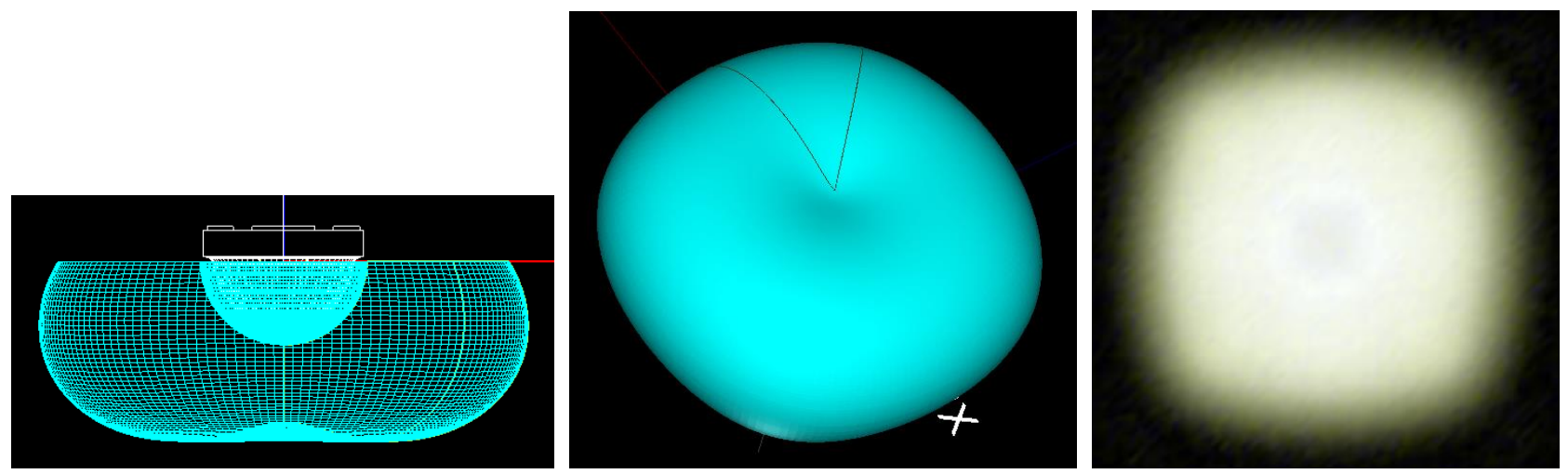

Figure 10. Free-form square beam lens design over a Cree XP-E2 LED. Lens cross section, 3D view of the outer surface and the resulting beam pattern on a horizontal plane below. 

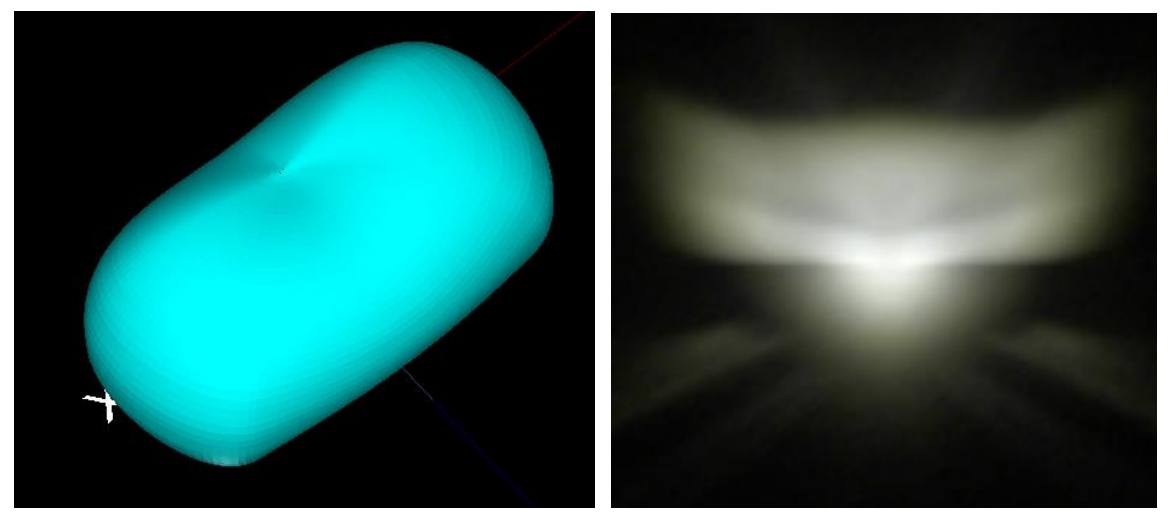

Figure 11. Free-form forward throw (type III) lens design that creates a rectangular beam pattern as required for roadway luminaires.

\subsection{Final project}

The final project allowed the students to select their design type, define the performance requirements and size constraints, and then design, fabricate and test the physical prototype. One design selected was a wall grazer that created a narrow beam that would uniformly illuminate a textured wall while highlighting the surface features. The design used a reflector optic since it could be 3D printed and used an adhesive, specular reflector material inside. A linear strip of LEDs was aimed up into the reflector, so all light coming out was under the control of the reflector. This design had some compromises in efficiency, which are acceptable for this product category where the wall appearance is the driving performance factor. Not all final project prototypes perform as expected since they will depend on the build quality, but those are important lessons to learn as well. Students are quite excited however, when their designs perform well like the one shown in figure 12 .
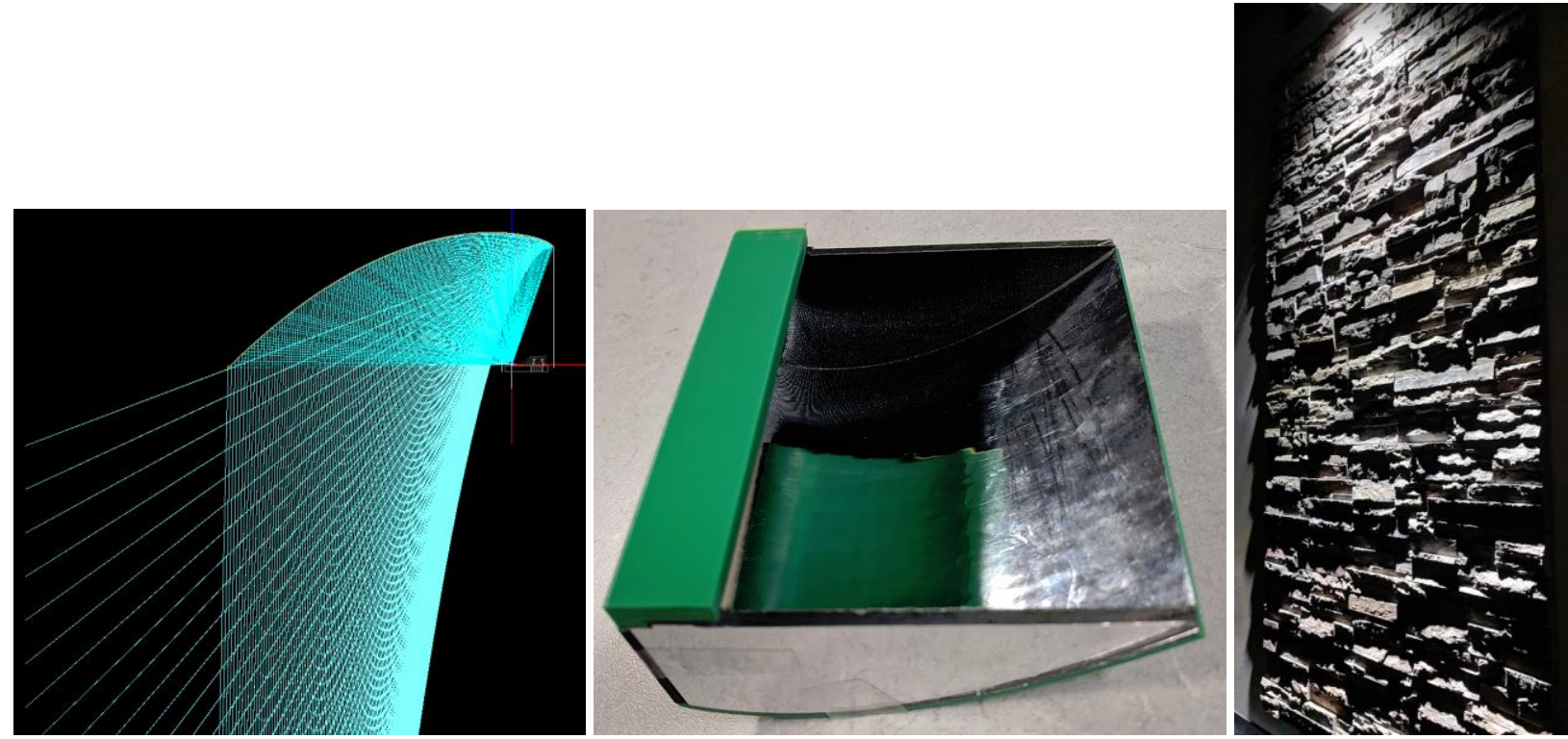

Figure 12. Wall grazer reflector aiming scheme shown on the left. The 3D printed prototype shown in the center and the working prototype lighting up a demo wall in the lighting lab. 